ASM Sc. J., 16, 2021

https://doi.org/10.32802/asmscj.2021.770

\title{
Development of Novel Subclasses for Bi- Univalent Functions
}

\author{
Munirah Rossdy ${ }^{1,2^{*}}$, Rashidah Omar ${ }^{2}$ and Shaharuddin Cik Soh ${ }^{1}$ \\ ${ }^{I}$ Faculty of Computer and Mathematical Sciences, Universiti Teknologi MARA, 40450, Shah Alam, Selangor, Malaysia \\ ${ }^{2}$ Faculty of Computer and Mathematical Sciences, Universiti Teknologi MARA Sabah Branch, 88997, Kota Kinabalu, \\ Sabah, Malaysia
}

\begin{abstract}
This manuscript presents the development of new subclasses for bi-univalent functions and the subclasses are closely related to Chebyshev polynomials having Al-Oboudi differential operator. The functions contained in the subclasses were used to account for the initial coefficient estimates of $\left|a_{2}\right|$ and $\left|a_{3}\right|$.
\end{abstract}

Keywords: coefficient estimates; subordination; Chebyshev Polynomials; bi-univalent; Al-Oboudi Operator

\section{INTRODUCTION}

Let $\mathbb{C}$ be a set of complex numbers, $\mathbb{R}=(-\infty, \infty)$, a set of real numbers and $\mathbb{N}:=\{1,2,3, \ldots\}=\mathbb{N}_{0} \backslash\{0\}$ representing a set of positive integers. Then, let $A$ be defined as $\Delta=$ $\{z: z \in \mathbb{C},|z|<1\}$, and an open unit disc denoted as a class function expressed in (1).

$$
f(z)=z+\sum_{k=2}^{\infty} a_{k} z^{k}
$$

The subclass of $A$ is represented by $S$, which is a normalised analytic function as shown in $f^{\prime}(0)=1$ and $f(0)=0$. Given that $K(\alpha)$ and $S^{*}(\alpha)$ are expressed as the convex and starlike functions respectively in the order of $\alpha(0 \leq \alpha<1)$, they can be represented by the renowned subclasses of $S$.

For $(f \in A)$, Al-Oboudi (2004) represented the operator as thus:

$D_{\delta}^{0} f(z)=f(z)$,

$D_{\delta}^{1} f(z)=(1-\delta) f(z)+\delta z f^{\prime}(z):=D_{\delta} f(z)(\delta \geq 0)$

$D_{\delta}^{n} f(z)=D_{\delta}\left(D_{\delta}^{n-1} f(z)\right)(n \in \mathbb{N})$

As $f$ is represented in (1), it can be seen from (2) and (3) that: $D_{\delta}^{n} f(z)=z+\sum_{k=2}^{\infty}[1+(k-1) \delta]^{n} a_{k} z^{k} \quad\left(n \in \mathbb{N}_{0}\right)$ where $D_{\delta}^{n} f(0)=0$. The Sălăgean's differential operator is obtained when

$\delta=1$. (Sălăgean, 1983).

A function $f: \Delta \rightarrow \mathbb{C}$ is called univalent on $\Delta$ (or schlict or one-to-one) if $f\left(z_{1}\right) \neq f\left(z_{2}\right)$ for all $z_{1}, z_{2} \in \Delta$ with $z_{1} \neq z_{2}$. Based on Duren (1983), the theorem of Koebe's one-quarter showed the images of $\Delta$ for every univalent function, $(f \in$ $S$ )enclosing $1 / 4$ radius disc. Hence, every inverse function of $f^{-1}$ in $(f \in A)$ can be defined as:

$$
\begin{gathered}
f^{-1}(f(z))=z \quad(z \in U), \\
f\left(f^{-1}(w)\right)=w \\
\left(|w|<r_{0}(f) ; r_{0}(f) \geq \frac{1}{4}\right) .
\end{gathered}
$$

Furthermore, $f^{-1}$ is shown as:

$$
\begin{aligned}
& g(w)=f^{-1}(w)=w-a_{2} w^{2}+\left(2 a_{2}^{2}-a_{3}\right) w^{3}- \\
& \left(5 a_{2}^{3}-5 a_{2} a_{3}+a_{4}\right) w^{4}+\cdots
\end{aligned}
$$

A function $(f \in A)$ is called bi-univalent in $\Delta$, if every of $f$ or $f^{-1}$ is univalent. Thus, the notation of bi-univalent functions class is expressed as $\sum$. The background and previous works on $\sum$ can be found in Srivastava et al. (2010) and Brannan and Taha $(1986 ; 1988)$. Indeed, the research 
findings of Srivastava et al. (2010) have been used as the basis of revitalisation for the research of numerous subclasses of biunivalent functions class $\Sigma$. Several researchers that conducted similar studies included: Aldawish et al. (2020), Khan et al. (2020), Hern and Janteng (2020), Omar et al. (2019), Porwal and Darus (2013), Bulut (2013), Çağlar et al. (2013), Hayami and Owa (2012), Xu et al. (2012a), Xu et al. (2012b) and Frasin and Aouf (2011).

Next, the concept of subordination was used, given that $f(z) \prec g(z)$, with $f$ being a subordinate to $g$, and both functions taken to be analytic. This implies that $f(z)=$ $g(w(z))$, where $w$ is taken as analytic in $\Delta$, which corresponds to $|w(z)|<1$ and $w(0)=0$.

Chebyshev polynomials applied in this study, is very relevant in numerical analysis. Chebyshev orthogonal polynomials are associated with important findings on $T_{n}(x)$ and $U_{n}(x)$ expressed as shown in (6).

$$
T_{n}(x)=\cos n \theta, \quad U_{n}(x)=\frac{\sin (n+1) \theta}{\sin \theta}
$$

where $(-1<x<1)$ is expressed as $x=\cos \theta$, and subscript $n$ indicated the degree of the polynomial. With the use of the function: $H(z, t)=\frac{1}{1-2 t z+z^{2}}$, we identified that when $t=\cos \alpha, \alpha \in\left(-\frac{\pi}{3}, \frac{\pi}{3}\right)$, thus, for all $z \in \Delta$,

$$
\begin{gathered}
H(z, t)=1+\sum_{n=1}^{\infty} \frac{\sin (n+1) \alpha}{\sin \alpha} z^{n}=1+2 \cos \alpha z+ \\
\left(3 \cos ^{2} \alpha-\sin ^{2} \alpha\right) z^{2}+\ldots
\end{gathered}
$$

hence, we have:

$$
\begin{gathered}
H(z, t)=1+U_{1}(t) z+U_{2}(t) z^{2}+\cdots \\
(z \in \Delta, t \in(-1,1))
\end{gathered}
$$

for $U_{n-1}=\frac{\sin (n \arccos t)}{\sqrt{1-t^{2}}}, n \in \mathbb{N}$ and equally

$$
\begin{gathered}
U_{n}(t)=2 t U_{n-1}(t)-U_{n-2}(t), \text { where: } \\
U_{1}(t)=2 t \\
U_{2}(t)=4 t^{2}-1, \\
U_{3}(t)=8 t^{3}-4 t, \ldots
\end{gathered}
$$

Next, $T_{n}(t)$ that generated the function is represented as:

$$
\sum_{n=0}^{\infty} T_{n}(t) z^{n}=\frac{1-t z}{1-2 t z+z^{2}}(z \in \Delta),
$$

with $t \in[-1,1]$. Nevertheless, $T_{n}(t)$ and $U_{n}(t)$ of the Chebyshev polynomials have the following relationships:

$$
\begin{gathered}
\frac{d T_{n}(t)}{d t}=n U_{n-1}(t), \\
T_{n}(t)=U_{n}(t)-t U_{n-1}(t), \\
2 T_{n}(t)=U_{n}(t)-U_{n-2}(t) .
\end{gathered}
$$

More details on the applications of Chebyshev polynomials can be found in the studies of Doha (1994) and Mason (1967). Inspired by the recent findings on the bi-univalent functions by Güney et al. (2017), Altinkaya and Yalcin (2016), Dziok et al. (2015), Murugusundaramoorthy et al. (2015), and Vijaya et al. (2014), we proposed the new subclasses of $\sum$ and determined the initial coefficients of $\left|a_{2}\right|$ and $\left|a_{3}\right|$ by the application of Chebyshev polynomials associated with $\mathrm{Al}-$ Oboudi differential operator.

\section{METHODS}

The main results of the subclasses were obtained by the application of the following definitions:

\section{Definition 1.}

For $0 \leq \lambda \leq 1, \delta \geq 0, n \in \mathbb{N}$ and $t \in(-1,1),(f \in \Sigma)$ of the form (1) is said to be in the class of $N_{\Sigma}^{\delta}[n, \lambda, H]$, if:

$(1-\lambda) \frac{D_{\delta}^{n+1} f(z)}{D_{\delta}^{n} f(z)}+\lambda \frac{D_{\delta}^{n+2} f(z)}{D_{\delta}^{n+1} f(z)} \prec H(z, t)$,

$(1-\lambda) \frac{D_{\delta}^{n+1} g(w)}{D_{\delta}^{n} g(w)}+\lambda \frac{D_{\delta}^{n+2} g(w)}{D_{\delta}^{n+1} g(w)} \prec H(w, t)$

where $D_{\delta}^{n}$ is the Al-Oboudi operator, $g$ as specified in (5) and $z, w \in \Delta$.

\section{Remark 1.}

The new subclasses of $\sum$ are introduced, by specifying the elements of $\lambda$ and $n$ in Definition 1 , taking $t \in(-1,1)$ and $f(z) \in \Sigma$.

(i) $N_{\Sigma}^{1}[n, 0, H] \equiv M_{\Sigma}^{k}(0, \Phi(z, t))$ (Güney et al., 2017)

(ii) $N_{\Sigma}^{1}[n, 1, H] \equiv M_{\Sigma}^{k}(1, \Phi(z, t))$ (Güney et al., 2017)

(iii) $N_{\Sigma}^{1}[0, \lambda, H] \equiv M_{\Sigma}^{0}(\lambda, \Phi(z, t))$ (Güney et al., 2017)

(iv) $N_{\Sigma}^{1}[0,0, H] \equiv M_{\Sigma}^{0}(0, \Phi(z, t))$ (Güney et al., 2017)

(v) $N_{\Sigma}^{1}[0,1, H] \equiv M_{\Sigma}^{0}(1, \Phi(z, t))$ (Güney et al., 2017) 


\section{Definition 2.}

For $0 \leq \beta \leq 1$ and $t \in(-1,1)$, a function, $f \in \Sigma$ as contained in (1) is said to be in the class $F_{\Sigma}^{\delta}[n, \beta, H]$,

if the following subordinations apply:

$$
\begin{gathered}
(1-\beta) \frac{D_{\delta}^{n} f(z)}{z}+\beta\left(D_{\delta}^{n} f(z)\right)^{\prime}<H(z, t), \\
(1-\beta) \frac{D_{\delta}^{n} g(w)}{w}+\beta\left(D_{\delta}^{n} g(w)\right)^{\prime} \prec H(w, t),
\end{gathered}
$$

$D_{\delta}^{n}$ is denoted as the Al-Oboudi operator, $g$ as expressed in (5) and $z, w \in \Delta$.

\section{Remark 2.}

Consequently, taking $t \in(-1,1)$ and $f(z) \in \Sigma$, and $\beta$ and $n$ as expressed in Definition 2, we have the following subclasses of $\sum$ as listed below:

(i) $F_{\Sigma}^{1}[n, 0, H] \equiv F_{\Sigma}^{k}(0, \Phi(z, t))$ (Güney et al., 2017)

(ii) $F_{\Sigma}^{1}[n, 1, H] \equiv F_{\Sigma}^{k}(1, \Phi(z, t))$ (Güney et al., 2017)

(iii) $F_{\Sigma}^{1}[0, \beta, H] \equiv F_{\Sigma}^{0}(\beta, \Phi(z, t))$ (Güney et al., 2017)

(iv) $F_{\Sigma}^{1}[0,0, H] \equiv F_{\Sigma}^{0}[0, \Phi(z, t)]$ (Güney et al., 2017)

(v) $F_{\Sigma}^{1}[0,1, H] \equiv F_{\Sigma}^{0}(1, \Phi(z, t))$ (Güney et al., 2017)

\section{RESULTS}

The main results of the subclasses are presented below:

\section{Theorem 1.}

Let $f$ expressed in (1) be in the class $N_{\Sigma}^{\delta}[n, \lambda, H]$ and $t \in$ $(0,1)$. Based on these:

$$
\left|a_{2}\right| \leq \frac{2 t \sqrt{2 t}}{\sqrt{\left[\begin{array}{l}
2 \delta(1+2 \lambda \delta)[1+2 \delta]^{n} \\
-\delta\left(\begin{array}{l}
\lambda^{2} \delta^{3}+3 \lambda \delta^{2} \\
+2 \lambda \delta+\delta+1
\end{array}\right)[1+\delta]^{2 n}
\end{array}\right] 4 t^{2}}}
$$

and,

$$
\left|a_{3}\right| \leq \frac{4 t^{2}}{\delta^{2}(1+\lambda \delta)^{2}[1+\delta]^{2 n}}+\frac{t}{\delta(1+2 \lambda \delta)[1+2 \delta]^{n}} .
$$

where $0 \leq \lambda \leq 1$.

Proof.

Utilising (8), we have:

$$
\begin{aligned}
& (1-\lambda) \frac{D_{\delta}^{n+1} f(z)}{D_{\delta}^{n} f(z)}+\lambda \frac{D_{\delta}^{n+2} f(z)}{D_{\delta}^{n+1} f(z)}=H(u(z), t), \\
& (1-\lambda) \frac{D_{\delta}^{n+1} g(w)}{D_{\delta}^{n} g(w)}+\lambda \frac{D_{\delta}^{n+2} g(w)}{D_{\delta}^{n+1} g(w)}=H(v(w), t) .
\end{aligned}
$$

$u(z)$ and $v(w)$ are denoted as:

$$
\begin{gathered}
u(z)=c_{1} z+c_{2} z^{2}+\ldots, \\
v(w)=d_{1} w+d_{2} w^{2}+\ldots
\end{gathered}
$$

where:

$$
\begin{aligned}
& |u(z)|<1, \\
& |v(w)|<1,
\end{aligned}
$$

$z \in \Delta$ and $u(0)=v(0)=0$ are analytic in $\Delta$. It is equally notable that:

$$
\begin{gathered}
|u(z)|=\left|c_{1} z+c_{2} z^{2}+\cdots\right|<1, \\
|v(w)|=\left|d_{1} w+d_{2} w^{2}+\ldots\right|<1,
\end{gathered}
$$

$z, w \in \Delta$,

where

$$
\begin{gathered}
\left|c_{j}\right| \leq 1,\left|d_{j}\right| \leq 1 \\
\forall j \in \mathbb{N} .
\end{gathered}
$$

Using (11) and (12) in (9) and (10), respectively, we have:

$$
\begin{aligned}
& (1-\lambda) \frac{D_{\delta}^{n+1} f(z)}{D_{\delta}^{n} f(z)}+\lambda \frac{D_{\delta}^{n+2} f(z)}{D_{\delta}^{n+1} f(z)} \\
& =1+U_{1}(t) u(z)+U_{2}(t) u^{2}(z)+\cdots,
\end{aligned}
$$

$$
\begin{aligned}
& (1-\lambda) \frac{D_{\delta}^{n+1} g(w)}{D_{\delta}^{n} g(w)}+\lambda \frac{D_{\delta}^{n+2} g(w)}{D_{\delta}^{n+1} g(w)} \\
& =1+U_{1}(t) v(w)+U_{2}(t) v^{2}(w)+\cdots
\end{aligned}
$$

In consideration of (1), (4), (5), (7) and (14), we have:

$$
\begin{aligned}
& 1+\delta(1+\lambda \delta)[1+\delta]^{n} a_{2} z \\
& +\left[\begin{array}{l}
2 \delta(1+2 \lambda \delta)[1+2 \delta]^{n} a_{3} \\
-\delta\left(1+2 \lambda \delta+\lambda \delta^{2}\right)[1+\delta]^{2 n} a_{2}^{2}
\end{array}\right] z^{2}+\cdots \\
& =1+U_{1}(t) c_{1}+\left[U_{1}(t) c_{2}+U_{2}(t) c_{1}^{2}\right] z^{2}+\cdots \\
& 1-\delta(1+\lambda \delta)[1+\delta]^{n} a_{2} w
\end{aligned}
$$

Thus:

$$
\begin{aligned}
& \quad \delta(1+\lambda \delta)[1+\delta]^{n} a_{2}=U_{1}(t) c_{1}, \\
& 2 \delta(1+2 \lambda \delta)[1+2 \delta]^{n} a_{3} \\
& -\delta\left(1+2 \lambda \delta+\lambda \delta^{2}\right)[1+\delta]^{2 n} a_{2}^{2} \\
& =U_{1}(t) c_{2}+U_{2}(t) c_{1}^{2} \\
& -\delta(1+\lambda \delta)[1+\delta]^{n} a_{2}=U_{1}(t) d_{1},
\end{aligned}
$$




$$
\begin{aligned}
& {\left[\begin{array}{l}
4 \delta(1+2 \lambda \delta)[1+2 \delta]^{n} \\
-\delta\left(1+2 \lambda \delta+\lambda \delta^{2}\right)[1+\delta]^{2 n}
\end{array}\right] a_{2}^{2} } \\
- & 2 \delta(1+2 \lambda \delta)[1+2 \delta]^{n} a_{3} \\
= & U_{1}(t) d_{2}+U_{2}(t) d_{1}^{2} .
\end{aligned}
$$

By utilising (15) and (17), we have:

$$
c_{1}=-d_{1}
$$

$2\left[\delta+\lambda \delta^{2}\right]^{2}[1+\delta]^{2 n} a_{2}{ }^{2}=U_{1}^{2}(t)\left[c_{1}^{2}+d_{1}^{2}\right]$.

By the addition of (16) and (18) and inputting into (20), gave rise to the below expression:

$$
a_{2}^{2}=\frac{U_{1}^{3}\left(c_{2}+d_{2}\right)}{2\left[\begin{array}{l}
\left\{\begin{array}{l}
2 \delta(1+2 \lambda \delta)[1+2 \delta]^{n} \\
-\delta\left(1+2 \lambda \delta+\lambda \delta^{2}\right)[1+\delta]^{2 n}
\end{array}\right\} U_{1}^{2}(t) \\
-\delta^{2}(1+\lambda \delta)^{2}[1+\delta]^{2 n} U_{2}(t)
\end{array}\right]}
$$

Taking $c_{2}$ and $d_{2}$, as expressed in (13) and inputting into (7), resulted to:

$$
\left|a_{2}\right| \leq \frac{2 t \sqrt{2 t}}{\sqrt{\left[\begin{array}{l}
2 \delta(1+2 \lambda \delta)[1+2 \delta]^{n} \\
\left.-\delta\left(\lambda^{2} \delta^{3}+3 \lambda \delta^{2}+2 \lambda \delta+\delta+1\right)[1+\delta]^{2 n}\right] \\
+\delta^{2}(1+\lambda \delta)^{2}[1+\delta]^{2 n}
\end{array}\right.}} .
$$

By the subtraction of (18) from (16) and inputting into (19) and (20), resulted to:

$$
a_{3}=\frac{U_{1}^{2}(t)\left(c_{1}^{2}+d_{1}^{2}\right)}{2 \delta^{2}(1+\lambda \delta)^{2}[1+\delta]^{2 n}}+\frac{U_{1}(t)\left(c_{2}-d_{2}\right)}{4 \delta(1+2 \lambda \delta)[1+2 \delta]^{n}}
$$

Using (7), and once again applying (13) to the coefficients $c_{1}, c_{2}, d_{1}$, and $d_{2}$, resulted to:

$$
\left|a_{3}\right| \leq \frac{4 t^{2}}{\delta^{2}(1+\lambda \delta)^{2}[1+\delta]^{2 n}}+\frac{t}{\delta(1+2 \lambda \delta)[1+2 \delta]^{n}}
$$

Based on theorem 5, the following corollaries were obtained.

\section{Corollary 1.}

Let $f$ be in the class $N_{\Sigma}^{\delta}[n, 0, H]$, then:

$$
\begin{gathered}
\left|a_{2}\right| \leq \frac{2 t \sqrt{2 t}}{\sqrt{\mid \begin{array}{l}
{\left[2 \delta[1+2 \delta]^{n}-\delta(\delta+1)[1+\delta]^{2 n}\right] 4 t^{2}} \\
+\delta^{2}[1+\delta]^{2 n}
\end{array}}} \\
\left|a_{3}\right| \leq \frac{4 t^{2}}{\delta^{2}[1+\delta]^{2 n}}+\frac{t}{\delta[1+2 \delta]^{n}} .
\end{gathered}
$$

Taken $\delta=1$, resulted to $M_{\Sigma}^{k}(0, \Phi(z, t))$ as introduced in theorem 5 (Güney et al., 2017).

\section{Corollary 2.}

Let $f$ be in the class $F_{\Sigma}^{\delta}[n, 1, H]$, then:

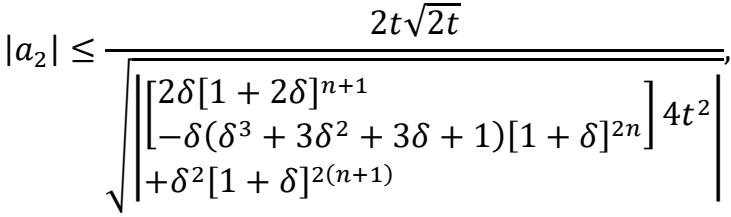

$$
\begin{aligned}
& \left|a_{3}\right| \leq \frac{4 t^{2}}{\delta^{2}[1+\delta]^{2(n+1)}}+\frac{t}{\delta[1+2 \delta]^{n+1}}
\end{aligned}
$$

Given $\delta=1$, resulted to $F_{\Sigma}^{k}(1, \Phi(z, t))$ as introduced in theorem 5 (Güney et al., 2017).

\section{Corollary 3.}

Let $f$ be in the class $N_{\Sigma}^{\delta}[0, \lambda, H]$, then:

$$
\begin{aligned}
& \left|a_{2}\right| \leq \frac{2 t \sqrt{2 t}}{\sqrt{\mid \begin{array}{l}
\delta^{2}(1+\lambda \delta)^{2} \\
-\delta\left(\delta^{3} \lambda^{2}+3 \lambda \delta^{2}-2 \lambda \delta+\delta-1\right) 4 t^{2} \mid
\end{array}}} \\
& \left|a_{3}\right| \leq \frac{4 t^{2}}{\delta^{2}(1+\lambda \delta)^{2}}+\frac{t}{\delta(1+2 \lambda \delta)}
\end{aligned}
$$

where $0 \leq \lambda \leq 1$.

Given $\delta=1$, resulted to $M_{\Sigma}^{0}(\lambda, \Phi(z, t))$ as introduced in theorem 5 (Güney et al., 2017).

\section{Corollary 4 .}

Taken $f$ to be in the class $N_{\Sigma}^{\delta}[0,0, H]$, then:

$$
\begin{gathered}
\left|a_{2}\right| \leq \frac{2 t \sqrt{2 t}}{\sqrt{\left|\delta^{2}-\delta(\delta-1) 4 t^{2}\right|}} \\
\left|a_{3}\right| \leq \frac{4 t^{2}}{\delta^{2}}+\frac{t}{\delta}
\end{gathered}
$$

Taken $\delta=1$, then, we had $M_{\Sigma}^{0}(0, \Phi(z, t))$ as introduced in theorem 5 (Güney et al., 2017).

\section{Corollary 5 .}

Taken $f$ to be in the class $N_{\Sigma}^{\delta}[0,1, H]$, then:

$$
\left|a_{2}\right| \leq \frac{2 t \sqrt{2 t}}{\sqrt{\left|\delta^{2}(1+\delta)^{2}-\delta\left(\delta^{3}+3 \delta^{2}-2 \delta+\delta-1\right) 4 t^{2}\right|}},
$$

and

$$
\left|a_{3}\right| \leq \frac{4 t^{2}}{\delta^{2}(1+\delta)^{2}}+\frac{t}{\delta(1+2 \delta)}
$$

Taken $\delta=1$, and $t \neq 1 / \sqrt{2}$, resulted to $M_{\Sigma}^{0}(1, \Phi(z, t))$ as introduced in theorem 5 (Güney et al., 2017). 


\section{Theorem 2.}

Taken $f$ as contained in (1) to be in the class $\left.F_{\Sigma}^{\delta}[n, \beta, H)\right]$ and $t \in(0,1)$ resulted to:

$$
\left|a_{2}\right| \leq \frac{2 t \sqrt{2 t}}{\sqrt{\mid \begin{array}{l}
\left.\mid(1+2 \beta)[1+2 \delta]^{n}-(1+\beta)^{2}[1+\delta]^{2 n}\right] 4 t^{2} \\
+(1+\beta)^{2}[1+\delta]^{2 n}
\end{array}}}
$$

and

$$
\left|a_{3}\right| \leq \frac{4 t^{2}}{(1+\beta)^{2}[1+\delta]^{2 n}}+\frac{2 t}{(1+2 \beta)[1+2 \delta]^{n}}
$$

\section{Proof.}

According to the proofs obtained in theorem (5), we acquired the succeeding expressions below:

$$
\begin{gathered}
(1+\beta)[1+\delta]^{n} a_{2}=U_{1}(t) c_{1}, \\
(1+2 \beta)[1+2 \delta]^{n} a_{3}=U_{1}(t) c_{2}+U_{2}(t) c_{1}^{2}, \\
-(1+\beta)[1+\delta]^{n} a_{2}=U_{1}(t) d_{1}, \\
2(1+2 \beta)[1+2 \delta]^{n} a_{2}^{2}-(1+2 \beta)[1+2 \delta]^{n} a_{3}= \\
U_{1}(t) d_{2}+U_{2}(t) d_{1}^{2} .
\end{gathered}
$$

From (23) and (25), it was established that:

$$
\begin{aligned}
c_{1} & =-d_{1}, \\
2(1+\beta)^{2}[1+\delta]^{2 n} a_{2}^{2} & =U_{1}^{2}(t)\left(c_{1}^{2}+d_{1}^{2}\right) .
\end{aligned}
$$

Then, the utilisation of (24), (26), and (28) resulted to:

$$
a_{2}^{2}=\frac{U_{1}^{3}(t)\left(c_{2}+d_{2}\right)}{2\left[\begin{array}{l}
(1+2 \beta)[1+2 \delta]^{n} U_{1}^{2}(t) \\
-(1+\beta)^{2}[1+\delta]^{2 n} U_{2}(t)
\end{array}\right]}
$$

Then, by the use of (7) and (13) for the coefficients $c_{2}$ and $d_{2}$, we obtained the sought-after bound on $\left|a_{2}\right|$ as stated in (21), and by subtracting (26) from (24), and inputting into (27) and (28), resulted to:

$$
a_{3}=\frac{U_{1}^{2}(t)\left(c_{1}^{2}+d_{1}^{2}\right)}{2(1+\beta)^{2}[1+\delta]^{2 n}}+\frac{U_{1}(t)\left(c_{2}-d_{2}\right)}{2(1+2 \beta)[1+2 \delta]^{n}} .
$$

Reiteratively, from the use of (7) and (13) for the coefficients $c_{1}, c_{2}, d_{1}$, and $d_{2}$, we got $\left|a_{3}\right|$ as contained in (22).

\section{Corollary 6.}

Given $f$ to be in the class $F_{\sum}^{\delta}[n, 0, H]$, resulted to:

$$
\left|a_{2}\right| \leq \frac{2 t \sqrt{2 t}}{\sqrt{\mid\left[[1+2 \delta]^{n}-[1+\delta]^{2 n}\right] 4 t^{2}+[1+\delta]^{2 n \mid}}}
$$

$$
\left|a_{3}\right| \leq \frac{4 t^{2}}{[1+\delta]^{2 n}}+\frac{2 t}{[1+2 \delta]^{n}} \text {. }
$$

$F_{\Sigma}^{1}[n, 0, H] \equiv F_{\Sigma}^{k}(0, \Phi(z, t))$

Taken $\delta=1$, we had $F_{\Sigma}^{k}(0, \Phi(z, t))$ as introduced in theorem 11 (Güney et al., 2017).

\section{Corollary 7 .}

Given $f$ to be in the class $F_{\Sigma}^{\delta}[n, 1, H]$, then:

$$
\begin{gathered}
\left|a_{2}\right| \leq \frac{2 t \sqrt{2 t}}{\sqrt{\left|\left[3[1+2 \delta]^{n}-4[1+\delta]^{2 n}\right] 4 t^{2}+4[1+\delta]^{2 n}\right|}} \\
\left|a_{3}\right| \leq \frac{t^{2}}{[1+\delta]^{2 n}}+\frac{2 t}{3[1+2 \delta]^{n}} .
\end{gathered}
$$

Taken $\delta=1$, resulted to $F_{\Sigma}^{k}(1, \Phi(z, t))$ as introduced in theorem 11 (Güney et al., 2017).

\section{SUMMARY}

The bi-univalent functions subclasses of $N_{\Sigma}^{\delta}[n, \lambda, H]$ and $\left.F_{\Sigma}^{\delta}[n, \beta, H)\right]$ were developed using the subordinations of Chebyshev polynomials, defined by the Al-Oboudi differential operator and by the subclasses applications, the coefficient estimate of $\left|a_{2}\right|$ and $\left|a_{3}\right|$ were determined. The bounds obtained in Theorem 1 and Theorem 2 are the best possible.

\section{REFERENCES}

Aldawish, I, Al-Hawary, T \& Frasin, BA 2020, 'Subclasses of Bi-univalent functions defined by Frasin differential operator', Mathematics, no. 8, pp. 783 .

Al-Oboudi, FM 2004, 'On univalent functions defined by a generalized Sălăgean operator', International Journal of
Mathematics and Mathematical Sciences, no. 27, pp. 14291436.

Altinkaya, S \& Yalcin, S 2016, 'On the Chebyshev polynomial bounds for classes of univalent functions', Khayyam Journal of Mathematics, vol. 2, no. 1, pp. 1-5. 
Brannan, DA \& Taha, TS 1986, 'On some classes of biunivalent functions', Studia Universitatis Babes-Bolyai, Mathematica, vol. 31, no. 2, pp. 70-77.

Brannan, DA \& Taha, TS 1988, 'On some classes of biunivalent functions', Mathematical Analysis and its applications, Mazhar S. M, Hamoui A, and Faour N. S, Eds., KFAS Proceedings Series, Pergamon Press (Elsevier Science Limited), Oxford, UK, no. 3, pp. 53-6o.

Bulut, S 2013, 'Coefficient estimates for a class of analytic and bi-univalent functions', Novi Sad Journal of Mathematics, vol. 43, no. 2, pp. 59-65.

Çağlar, M, Orhan, H \& Yağmur, N 2013, 'Coefficient bounds for new subclasses of bi-univalent functions', Filomat, vol. 27, no. 7, pp. 1165-1171.

Doha, EH 1994, 'The first and second kind chebyshev coefficients of the moments for the general order derivative on an infinitely differentiable function', International Journal of Computer Mathematics, vol. 51, no. 1-2, pp. 2135 .

Duren, PI 1983, 'Univalent functions', Grundlehren der Mathematischen Wissenschaften, Springer, New York USA, pp. 259.

Dziok, J, Raina, RK \& Sokol, J 2015, 'Application of Chebyshev polynomials to classes of analytic functions', Comptes Rendus Mathematique, vol. 353, no. 5, pp. 433438, 2015 .

Frasin, BA \& Aouf, MK 2011, 'New subclasses of bi-univalent functions', Applied Mathematics Letters, vol. 24, no. 9, pp. 1569-1573.

Güney, HÖ, Murugusundaramoorthy, G \& Vijaya, K 2017, 'Coefficient Bounds for Subclasses of Biunivalent Functions Associated with the Chebyshev Polynomials,' Journal of Complex Analysis, Article ID 4150210, pp. 1-7.

Hayami, T \& Owa, S 2012, 'Coefficient bounds for biunivalent functions, Panamerican Mathematical Journal, vol. 22, no. 4, pp. 15-26.

Hern, ALP \& Janteng, A 2020, 'Some Coefficient Problems On Bi-Univalent Functions', ASM Sc. J., vol. 13.

Khan, B, Srivastava, HM, Tahir, M, Darus, M, Ahmad, QZ \& Khan, N 2020, 'Applications of a certain $q$ - integral operator to the subclasses of analytic and bi-univalent functions', AIMS Mathematics, vol. 6, no. 1, pp. 1024-1039. Mason, JC 1967, 'Chebyshev polynomial approximations for the L-membrane eigenvalue problem', SIAM Journal on Applied Mathematics, vol. 15, pp. 172-186.

Murugusundaramoorthy, G, Selvaraj, C \& Babu, OS 2015, 'Coefficient Estimates For Pascu-type Subclasses of Bi-
Univalent functions based on subordination', International Journal of Nonlinear Science, vol. 19, no. 1, pp. 47-52.

Omar, R, Rossdy, M \& Halim, SA 2019, 'Feketo-Szegö inequalities for certain subclasses of Bi-Univalent functions', Journal of Physics: Conference Series, 1212 012006.

Porwal, S \& Darus, M 2013, 'On a new subclass of bi-univalent functions', Journal of the Egyptian Mathematical Society, vol. 21, no. 3, pp. 190-193.

Sălăgean, GS 1983, 'Subclasses of univalent functions', in Proceedings of the $5^{\text {th }}$ Romanian Finnish Seminar in Complex Analysis, Bucharest, Romania, pp. 362-372.

Srivasta, HM, Mishra, AK \& Gochhayat, P 2010, 'Certain subclasses of analytic and bi-univalent functions', Applied Mathematics Letters, vol. 23, no. 10, pp. 1188-1192.

Vijaya, K, Kasthuri, M \& Murugusundaramoorthy, G 2014, 'Coefficient Bounds for Subclasses of Biunivalent Functions Defined By The Sălăgean Derivative Operator', Boletin de la Asociaciton Matematica Venezolana.

$\mathrm{Xu}, \mathrm{Q}-\mathrm{H}$, Gui, Y-C \& Srivastava, HM 2012a, 'Coefficient estimates for a certain subclass of analytic and bi-univalent functions', Applied Mathematics Letters, vol. 25, no. 6, pp. 990-994.

Xu, Q-H, Xiao, H-G \& Srivastava, HM 2012b, 'A certain general subclass of analytic and bi-univalent functions and associated coefficient estimate problems', Applied Mathematics and Computation, vol. 218, no. 23, pp. 1146111465 . 